\title{
Dynamic Behaviors of an SEIR Epidemic Model in a Periodic Environment with Impulse Vaccination
}

\author{
Mei Yan ${ }^{1,2}$ and Zhongyi Xiang ${ }^{1,2}$ \\ ${ }^{1}$ Key Laboratory of Biologic Resources Protection and Utilization of Hubei Province, Enshi, Hubei 445000, China \\ ${ }^{2}$ Department of Mathematics, Hubei Institute for Nationalities, Enshi, Hubei 445000, China \\ Correspondence should be addressed to Zhongyi Xiang; zhyxiang260@yahoo.com.cn
}

Received 20 April 2013; Revised 27 December 2013; Accepted 30 December 2013; Published 23 February 2014

Academic Editor: Aura Reggiani

Copyright (c) 2014 M. Yan and Z. Xiang. This is an open access article distributed under the Creative Commons Attribution License, which permits unrestricted use, distribution, and reproduction in any medium, provided the original work is properly cited.

\begin{abstract}
We consider a nonautonomous SEIR endemic model with saturation incidence concerning pulse vaccination. By applying Floquet theory and the comparison theorem of impulsive differential equations, a threshold parameter which determines the extinction or persistence of the disease is presented. Finally, numerical simulations are given to illustrate the main theoretical results and it shows that pulse vaccination plays a key role in the disease control.
\end{abstract}

\section{Introduction}

Millions of human beings suffer or die of various infectious diseases every year. Infectious diseases such as measles, polio, diphtheria, tetanus, and pertussis have tremendous influence on human life. Controlling infectious diseases has been an increasingly complex issue worldwide in recent years. Pulse vaccination is an effective method to use in controlling the transmission of diseases which has gained prominence. Epidemiological models with pulse vaccination have been set up and investigated in many literatures [1-8]. This kind of vaccination strategy is usually called pulse vaccination strategy (PVS). This vaccination is called impulsive when all the vaccine doses are applied in a very short span of time.

As we all known, some diseases may be governed directly or indirectly by environmental factors such as temperature, humidity, and barometric pressure; see [9-13], for example. It is realistic to investigate this kind of epidemic models with periodic (seasonal) fluctuations. Bai and Zhou [14] studied the existence and the number of periodic solutions in an SIR epidemic model with seasonal contact rate. One of the most important subjects in this field is to obtain a threshold that determines the persistence or extinction of a diseases. According to $[15,16]$, we know that the basic reproduction number for the periodic epidemic models is generally different from the basic reproduction number of the tim-averaged autonomous system. They defined the basic reproduction number which is a threshold between the extinction and the uniform persistence of the disease. In [17], Nakata and Kuniya considered the following SEIRS epidemic model:

$$
\begin{gathered}
\dot{S}(t)=\lambda(t)-\mu(t) S(t)-\beta(t) S(t) I(t)+\delta(t) R(t), \\
\dot{E}(t)=\beta(t) S(t) I(t)-(\mu(t)+\varepsilon(t)) E(t), \\
\dot{I}(t)=\varepsilon(t) E(t)-(\mu(t)+\gamma(t)) I(t), \\
\dot{R}(t)=\gamma(t) I(t)-(\mu(t)+\delta(t)) R(t),
\end{gathered}
$$

where $S(t), E(t), I(t)$, and $R(t)$ denote susceptible, exposed (infected but not infectious), infectious, and recovered population at time $t \geq 0$, respectively. $\lambda(t), \mu(t), \beta(t), \delta(t), \varepsilon(t)$, and $\gamma(t)$ are continuous, positive $\omega$-periodic functions.

Many epidemic models with constant coefficients and pulse vaccination have been proposed and studied to understand mechanism of disease transmission. However, there have been less results on the pulse vaccination models with variable coefficients. Zhang and Teng [18] formulated a nonautonomous SEIRS model in epidemiology. Under the quite weak assumptions, they establish some sufficient conditions to prove the permanence and extinction of disease. The nonautonomous models are more realistic. On the basis of the above discussion, we formulate the following SEIR epidemic 
model which takes into account both the saturation incidence and the effects of pulse vaccination:

$$
\begin{array}{r}
\dot{S}(t)=\lambda(t)-\mu(t) S(t)-\frac{\beta(t) S(t) I(t)}{1+\alpha(t) S(t)}, \\
\dot{E}(t)=\frac{\beta(t) S(t) I(t)}{1+\alpha(t) S(t)}-(\mu(t)+\varepsilon(t)) E(t), \\
\dot{I}(t)=\varepsilon(t) E(t)-(\mu(t)+\gamma(t)) I(t), \\
\dot{R}(t)=\gamma(t) I(t)-\mu(t) R(t), \\
t \neq k T, k \in \mathbb{N}, \\
S\left(t^{+}\right)=\left(1-\theta_{k}\right) S(t), \\
E\left(t^{+}\right)=E(t), \\
I\left(t^{+}\right)=I(t), \\
R\left(t^{+}\right)=R(t)+\theta_{k} S(t), \\
t=k T, k \in \mathbb{N},
\end{array}
$$

where $\lambda(t), \mu(t), \beta(t), \varepsilon(t)$, and $\gamma(t)$ are continuous, positive $\omega$-periodic functions. $\beta(t) S(t) /(1+\alpha(t) S(t))$ is the saturation incidence. The function $\lambda(t)$ is the recruitment rate of the susceptible population and $\mu(t)$ is the death rate of the population. $\beta(t)$ is the transmission coefficient and $\varepsilon(t)$ and $\gamma(t)$ are the instantaneous per capita rates of leaving the latent stage and infected stage, respectively. $T$ is the constant period between two pulse vaccinations, $\theta_{k}\left(0<\theta_{k}<1\right)$ is the fraction of susceptible to whom the vaccination is inoculated at times $t=k T, q=\omega / T$ is a positive integer, and $\theta_{k}=\theta_{k+q}$, $k \in \mathbb{N}$.

This paper is organized as follows. In Section 2, sufficient conditions for the global attractivity of disease-free periodic solution of system (2) are obtained. In Section 3, the permanence of disease is discussed. Numerical analysis and briefly discuss are given in the last Section.

\section{Stability of the Disease-Free Periodic Solution}

In the section, we will study the global asymptotical stability of the disease-free periodic solution of system (2). Before staring our theorem, we give the following lemma.

Lemma 1. Consider the following system:

$$
\begin{array}{r}
\dot{S}(t)=\lambda(t)-\mu(t) S(t), \\
\dot{R}(t)=-\mu(t) R(t), \\
t \neq k T, k \in \mathbb{N}, \\
S\left(t^{+}\right)=\left(1-\theta_{k}\right) S(t), \\
R\left(t^{+}\right)=R(t)+\theta_{k} S(t), \\
t=k T, k \in \mathbb{N} .
\end{array}
$$

Then system (3) has a unique positive periodic solution

$$
\begin{aligned}
S^{*}(t)= & \left(\prod_{i=1}^{v}\left(1-\theta_{i}\right)\right) \exp \left(-\int_{n \omega}^{t} \mu(x) d x\right) S^{*} \\
& +\sum_{j=1}^{v}\left(\prod_{i=j}^{v}\left(1-\theta_{i}\right)\right) \exp \left(-\int_{0}^{t} \mu(x) d x\right) \\
& \times \int_{n \omega+(j-1) T}^{n \omega+j T} \exp \left(-\int_{0}^{x} \mu(y) d y\right) \lambda(x) d x \\
& +\exp \left(-\int_{0}^{t} \mu(x) d x\right) \\
& \times \int_{n \omega+v T}^{t} \exp \left(-\int_{0}^{x} \mu(y) d y\right) \lambda(x) d x, \\
R^{*}(t)= & \exp \left(-\int_{n \omega}^{t} \mu(x) d x\right) R^{*} \\
& +\sum_{i=1}^{v} \theta_{i} \exp \left(-\int_{n \omega+i T}^{t} \mu(x) d x\right) S^{*}(n \omega+i T), \\
n \omega+v T<t \leq n \omega+(v+1) T, & v=0,1, \ldots, q-1, \quad n \in \mathbb{N},
\end{aligned}
$$

which is globally asymptotically stable, where

$$
\begin{aligned}
S^{*}= & \frac{1}{1-}\left(\prod_{i=1}^{q}\left(1-\theta_{i}\right)\right) \exp \left(-\int_{n \omega}^{(n+1) \omega} \mu(x) d x\right) \\
& \times\left[\sum_{j=1}^{q-1}\left(\prod_{i=j}^{q}\left(1-\theta_{i}\right)\right) \exp \left(-\int_{0}^{(n+1) \omega} \mu(x) d x\right)\right. \\
& \times \int_{n \omega+(j-1) T}^{n \omega+j T} \exp \left(-\int_{0}^{x} \mu(y) d y\right) \lambda(x) d x \\
& +\left(1-\theta_{q}\right) \exp \left(-\int_{0}^{(n+1) \omega} \mu(x) d x\right) \\
R^{*}= & \left.\times \int_{n \omega+v T}^{(n+1) \omega} \exp \left(-\int_{0}^{x} \mu(y) d y\right) \lambda(x) d x\right] \\
& 1-\exp \left(-\int_{n \omega}^{t} \mu(x) d x\right) R\left(n \omega^{+}\right) \\
& \times \sum_{i=1}^{q-1} \theta_{i} \exp \left(-\int_{n \omega+i T}^{(n+1) \omega} \mu(x) d x\right) S(n \omega+i T) \\
& +\theta_{q} S((n+1) \omega) .
\end{aligned}
$$


Proof. By calculating, we obtain the solution of system (3) with respect to $t \in(n \omega+v T, n \omega+(v+1) T]$ and $v=0,1, \ldots, q-$ $1, n \in \mathbb{N}$,

$$
\begin{aligned}
S(t)= & \left(\prod_{i=1}^{v}\left(1-\theta_{i}\right)\right) \exp \left(-\int_{n \omega}^{t} \mu(x) d x\right) S\left(n \omega^{+}\right) \\
& +\sum_{j=1}^{v}\left(\prod_{i=j}^{v}\left(1-\theta_{i}\right)\right) \exp \left(-\int_{0}^{t} \mu(x) d x\right) \\
& \times \int_{n \omega+(j-1) T}^{n \omega+j T} \exp \left(-\int_{0}^{x} \mu(y) d y\right) \lambda(x) d x \\
& +\exp \left(-\int_{0}^{t} \mu(x) d x\right) \\
& \times \int_{n \omega+v T}^{t} \exp \left(-\int_{0}^{x} \mu(y) d y\right) \lambda(x) d x, \\
R(t)= & \exp \left(-\int_{n \omega}^{t} \mu(x) d x\right) R\left(n \omega^{+}\right) \\
& +\sum_{i=1}^{v} \theta_{i} \exp \left(-\int_{n \omega+i T}^{t} \mu(x) d x\right) S(n \omega+i T) .
\end{aligned}
$$

Set $M_{n}=S\left(n \omega^{+}\right)$. From (6) and $q T=\omega$, we have

$$
\begin{aligned}
M_{n+1}= & S\left((n+1) \omega^{+}\right)=S\left(n \omega+q T^{+}\right) \\
= & \left(\prod_{i=1}^{q}\left(1-\theta_{i}\right)\right) \exp \left(-\int_{n \omega}^{(n+1) \omega} \mu(x) d x\right) M_{n} \\
& +\sum_{j=1}^{q-1}\left(\prod_{i=j}^{q}\left(1-\theta_{i}\right)\right) \exp \left(-\int_{0}^{(n+1) \omega} \mu(x) d x\right) \\
& \times \int_{n \omega+(j-1) T}^{n \omega+j T} \exp \left(-\int_{0}^{x} \mu(y) d y\right) \lambda(x) d x \\
& +\left(1-\theta_{q}\right) \exp \left(-\int_{0}^{(n+1) \omega} \mu(x) d x\right) \\
& \times \int_{n \omega+v T}^{(n+1) \omega} \exp \left(-\int_{0}^{x} \mu(y) d y\right) \lambda(x) d x \\
\triangleq & f\left(M_{n}\right),
\end{aligned}
$$

where $f$ is the stroboscopic map. It is easy to know that the map has the unique positive fix point which implies that the unique periodic solution $S^{*}(t)$ of system (3) is globally asymptotically stable. Similarly, we can obtain that the periodic solution $R^{*}(t)$ of system (3) is unique and globally asymptotically stable. The proof is completed.

We will present the Floquet theory for the following linear $T$-periodic impulsive equation:

$$
\begin{gathered}
\frac{d x}{d t}=A(t) x, \quad t \neq \tau_{k}, \quad t \in R, \\
\Delta x=B_{k} x, \quad t=\tau_{k}, \quad k \in Z .
\end{gathered}
$$

Introduce the following conditions:

$H_{1}: A(\cdot) \in \operatorname{PC}\left(R, C^{n \times n}\right)$ and $A(t+T)=A(t)(t \in R)$,

$H_{2}: B_{k} \in C^{n \times n}, \operatorname{det}\left(E+B_{k}\right) \neq 0, \tau_{k}<\tau_{k+1}(k \in Z)$,

$H_{3}$ : there exists a $q \in N$, such that $B_{k+q}=B_{k}, \tau_{k+q}=$ $\tau_{k}+T(k \in Z)$.

Note. PC means the set of functions which are piecewise continuous.

Let $\Phi(t)$ be a fundamental matrix of (9); then there exists a unique nonsingular matrix $M \in C^{n \times n}$ such that

$$
\Phi(t+T)=\Phi(t) M \quad(t \in R) .
$$

By equality (10) there corresponds to the fundamental matrix $\Phi(t)$ the constant matrix $M$ which we call the monodromy matrix of (9). All monodromy matrices of (9) are similar and have the same eigenvalues. The eigenvalues $\mu_{1}, \mu_{2}, \ldots, \mu_{n}$ of the monodromy matrices are called the Floquet multipliers of (9).

Lemma 2 (see [19, Chapter 2, Theorem 3.5] (Floquet theory)). Let conditions $\left(\mathrm{H}_{1}-\mathrm{H}_{3}\right)$ hold. Then the linear T-periodic impulsive equation (9) is

(1) stable if and only if all multipliers $\mu_{j}(j=1,2, \ldots, n)$ of (9) satisfy the inequality $\left|\mu_{j}\right| \leq 1$ and, moreover, to those $\mu_{j}$ for which $\left|\mu_{j}\right|=1$ correspond simple elementary divisors;

(2) asymptotically stable if and only if all multipliers $\mu_{j}(j=1,2, \ldots, n)$ of $(9)$ satisfy the inequality $\left|\mu_{j}\right|<$ 1 ;

(3) unstable if $\left|\mu_{j}\right|>1$ for some $j=1,2, \ldots, n$.

Let $\left(\mathbb{R}^{j}, \mathbb{R}_{+}^{j}\right)$ be the standard ordered $j$-dimensional Euclidean space with a norm $\|\cdot\|$. For $u, v \in \mathbb{R}^{j}$, we write $u \geq v$ provided $u-v \in \mathbb{R}^{j}, u \gg v$ provided $u-v \in \operatorname{Int}\left(\mathbb{R}^{j}\right)$, respectively.

Let $A(t)$ on the interval $((k-1) T, k T](k \in \mathbb{N})$ be a bounded, continuous, cooperative, and irreducible $j \times j$ matrix function and $A(t)=A(\omega+t)$. We consider the following system:

$$
\begin{gathered}
\frac{d x}{d t}=A(t) x, \\
x(k T)=x\left(k T^{+}\right), \quad k \in \mathbb{N} .
\end{gathered}
$$

Let $\Phi_{A}(t)$ be the fundamental solution matrix of (11), and let $r\left(\Phi_{A}(\omega)\right)$ be the spectral radius of $\Phi_{A}(\omega)$. By the PerronFrobenius theorem, $r\left(\Phi_{A}(\omega)\right)$ is the principle eigenvalue of $\Phi_{A}(\omega)$ in the sense that it is simple and admits an eigenvector $\nu^{*} \gg 0$. By the similar proof of Lemma 2.1 in [17], we have the following result.

Lemma 3. Denote $\mu=(1 / \omega) \ln r\left(\Phi_{A}(\omega)\right)$. Then there exists a positive, $\omega$-periodic function $v(t)$ such that $e^{\mu t} v(t)$ is a solution of (11). 
Define

$$
\begin{aligned}
F & =\left[\begin{array}{cc}
0 & \frac{\beta(t) S^{*}(t)}{1+\alpha(t) S^{*}(t)} \\
\varepsilon(t) & 0
\end{array}\right], \\
V(t) & =\left[\begin{array}{cc}
\mu(t)+\varepsilon(t) & 0 \\
0 & \mu(t)+\gamma(t)
\end{array}\right] .
\end{aligned}
$$

Theorem 4. If $r\left(\Phi_{F-V}(\omega)\right)<1$, then the disease-free period solution $\left(S^{*}(t), 0,0, R^{*}(t)\right)$ of system $(2)$ is globally asymptotically stable.

Proof. Firstly, we demonstrate the local stability of the disease-free period solution $\left(S^{*}(t), 0,0, R^{*}(t)\right)$. Define $S(t)=$ $u_{1}(t)+S^{*}(t), E(t)=u_{2}(t), I(t)=u_{3}(t)$, and $R(t)=u_{4}(t)+$ $R^{*}(t)$. Then (2) can be expanded in a Taylor series. After neglecting higher order terms, the linearized equations can be written as

$$
\begin{gathered}
\dot{V}_{1}(t)=A_{1}(t) V_{1}(t), \quad t \neq k T, k \in \mathbb{N}, \\
V_{1}\left(t^{+}\right)=B_{1}(t) V_{1}(t), \quad t=k T, k \in \mathbb{N},
\end{gathered}
$$

$$
M=\left[\begin{array}{ccc}
\prod_{k=1}^{q}\left(1-\theta_{k}\right) \exp \left(-\int_{0}^{\omega} \mu(\tau) d \tau\right) & * & 0 \\
0 & \Phi_{F-V}(\omega) & 0 \\
0 & * & \exp \left(-\int_{0}^{\omega} \mu(\tau) d \tau\right)
\end{array}\right] .
$$

There is no need to calculate the exact form of $*$ as it is not required in the following analysis. The stability of the period solution $\left(S^{*}(t), 0,0, R^{*}(t)\right)$ is determined by the eigenvalues of $M$. In view of Lemma 2, the disease-free period solution $\left(S^{*}(t), 0,0, R^{*}(t)\right)$ of system (2) is local stable if $r\left(\Phi_{F-V}(\omega)\right)<$ 1 .

Secondly, we will prove the global attractivity of the disease-free period solution. According to the first equation of system (2) and the positivity of the solutions, we have

$$
\begin{gathered}
\dot{S}(t) \leq \lambda(t)-\mu(t) S(t), \quad t \neq k T, k \in \mathbb{N} \\
S\left(t^{+}\right)=\left(1-\theta_{k}\right) S(t), \quad t=k T, k \in \mathbb{N}
\end{gathered}
$$

Consider the following auxiliary system:

$$
\begin{gathered}
\dot{x}(t)=\lambda(t)-\mu(t) x(t), \quad t \neq k T, k \in \mathbb{N}, \\
x\left(t^{+}\right)=\left(1-\theta_{k}\right) x(t), \quad t=k T, k \in \mathbb{N} .
\end{gathered}
$$

Lemma 1 implies that system (17) admits a positive periodic solution $S^{*}(t)$ which is globally asymptotically stable. From the comparison theorem in impulsive differential equations, where $V_{1}(t)=\left(u_{1}(t), u_{2}(t), u_{3}(t), u_{4}(t)\right)^{T}$ and

$$
\begin{aligned}
& A_{1}(t) \\
& =\left[\begin{array}{cccc}
-\mu(t) & 0 & -\frac{\beta(t) S^{*}(t)}{1+\alpha(t) S^{*}(t)} & 0 \\
0 & -(\mu(t)+\varepsilon(t)) & \frac{\beta(t) S^{*}(t)}{1+\alpha(t) S^{*}(t)} & 0 \\
0 & \varepsilon(t) & -(\mu(t)+\gamma(t)) & 0 \\
0 & 0 & \\
& \\
& B_{1}(t)=\left[\begin{array}{cccc}
1-\theta_{k} & 0 & 0 & 0 \\
0 & 1 & 0 & 0 \\
0 & 0 & 1 & 0 \\
\theta_{k} & 0 & 0 & 1
\end{array}\right] .
\end{array}\right],
\end{aligned}
$$

Thus the monodromy matrix of system (13) is

we have $S(t) \leq x(t)$. Hence, for any $\epsilon>0$, there exits $T_{1}>0$ such that $S(t) \leq S^{*}(t)+\epsilon$ for $t>T_{1}$. Then it holds that

$$
\begin{gathered}
\dot{E}(t) \leq \frac{\beta(t)\left(S^{*}(t)+\epsilon\right) I(t)}{1+\alpha(t)\left(S^{*}(t)+\epsilon\right)}-(\mu(t)+\varepsilon(t)) E(t), \\
\dot{I}(t)=\varepsilon(t) E(t)-(\mu(t)+\gamma(t)) I(t),
\end{gathered}
$$

for $t>T_{1}$. Put $F_{1}(t)$ be $2 \times 2$ matrix function such that

$$
F_{1}(t)=\left[\begin{array}{cc}
-(\mu(t)+\varepsilon(t)) & \frac{\beta(t)\left(S^{*}(t)+\epsilon\right) I(t)}{1+\alpha(t)\left(S^{*}(t)+\epsilon\right)} \\
\varepsilon(t) & -(\mu(t)+\gamma(t))
\end{array}\right]
$$

Since $r\left(\Phi_{F-V}(\omega)\right)<1$ and $r\left(\Phi_{F_{1}}(\omega)\right)$ is continuous for small $\epsilon$, we can choose $\epsilon$ sufficiently small so that $r\left(\Phi_{F_{1}}(\omega)\right)<1$.

Consider the following system:

$$
\begin{gathered}
\frac{d x}{d t}=F_{1}(t) x, \\
x(k T)=x\left(k T^{+}\right), \quad k \in \mathbb{N} .
\end{gathered}
$$

By Lemma 3 and the comparison principle, there exists a positive $\omega$-periodic function $v_{1}(t)$ such that $J(t) \leq v_{1}(t) \exp \left(p_{1} t\right)$ where $J(t)=(E(t), I(t))^{T}$ and $p_{1}=(1 / \omega) \ln r\left(\Phi_{F_{1}}(\omega)\right)<0$. Then we get that $\lim _{t \rightarrow+\infty} E(t)=0$ and $\lim _{t \rightarrow+\infty} I(t)=0$. 
From the first and third equations of system (2), Lemma 1 and the limiting system theory, we have $\lim _{t \rightarrow+\infty}\left(S(t)-S^{*}(t)\right)=0$ and $\lim _{t \rightarrow+\infty}\left(R(t)-R^{*}(t)\right)=0$. This completes the proof.

\section{Permanent}

Next, we give the conditions which assure that the disease is endemic (i.e., the disease is permanent) if the infectious population persists above a certain positive level for large enough time. Let $\lambda^{L}=\max _{0 \leq t \leq \omega} \lambda(t), \beta^{M}=\min _{0 \leq t \leq \omega} \beta(t)$, $\beta^{L}=\max _{0 \leq t \leq \omega} \beta(t), \alpha^{M}=\min _{0 \leq t \leq \omega} \alpha(t), \alpha^{L}=\max _{0 \leq t \leq \omega} \alpha(t)$, $\mu^{M}=\min _{0 \leq t \leq \omega} \mu(t), \mu^{L}=\max _{0 \leq t \leq \omega} \mu(t), \varepsilon^{M}=\min _{0 \leq t \leq \omega} \varepsilon(t)$, $\varepsilon^{L}=\max _{0 \leq t \leq \omega} \varepsilon(t), \gamma^{M}=\min _{0 \leq t \leq \omega} \gamma(t)$, and $\theta^{L}=$ $\max _{0 \leq k \leq q} \theta_{k}$. Using the same discussions as those in the proof of Lemma 2.2 in [17], we have

$$
\limsup _{t \rightarrow+\infty} I(t) \leq \frac{\lambda^{L}}{\mu^{M}} .
$$

With the similarly method of Theorem 6 see also in [20], we have the following lemma.

Lemma 5. The susceptible population is bounded away from zero so that $S(t) \geq S^{M}$ for sufficiently large $t$, where

$$
\begin{aligned}
S^{M}= & \left(\lambda^{M}\left(1-\theta^{L}\right)\left(1-\exp \left(-\left(\beta^{L}\left(\lambda^{L} / \mu^{M}\right)+\mu^{M}\right) T\right)\right)\right) \\
& \times\left(\left(\beta^{L}\left(\lambda^{L} / \mu^{M}\right)+\mu^{M}\right)\left(1-\theta^{L}\right)\right. \\
& \left.\quad \times\left(1-\left(1-\theta^{L}\right) \exp \left(-\left(\beta^{L}\left(\lambda^{L} / \mu^{M}\right)+\mu^{M}\right) T\right)\right)\right)^{-1} \\
& -\varepsilon^{1}, \quad\left(\varepsilon^{1} \text { is sufficiently small }\right) .
\end{aligned}
$$

\section{Define}

$$
F_{2}(t)=\left[\begin{array}{cc}
-(\mu(t)+\varepsilon(t)) & \frac{\beta(t)\left(S^{*}(t)-\epsilon\right) I(t)}{1+\alpha(t)\left(S^{*}(t)-\epsilon\right)} \\
\varepsilon(t) & -(\mu(t)+\gamma(t))
\end{array}\right] .
$$

We consider following system:

$$
\begin{gathered}
\frac{d x}{d t}=F_{2}(t) x, \\
x(k T)=x\left(k T^{+}\right), \quad k \in \mathbb{N} .
\end{gathered}
$$

Define $p_{2}=(1 / \omega) \ln \Phi_{F_{2}}(\omega)$. By Lemma 3, there exist a positive, $\omega$-periodic function $v_{2}(t)$ such that $v_{2}(t) \exp \left(p_{2} t\right)$ is a solution of $(24)$, where $v_{2}(t)=\left(v_{21}(t), v_{22}(t)\right)^{T}$.

Theorem 6. Assume that $r\left(\Phi_{F-V}(\omega)\right)>1$ holds; then there exists a positive constant $m_{0}$, such that, for all $(S(0), E(0)$, $I(0), R(0))=\left(S^{0}, E^{0}, I^{0}, R^{0}\right) \in \mathbb{R}_{+}^{2} \times \operatorname{Int}\left(\mathbb{R}_{+}\right) \times \mathbb{R}_{+}$, the solution $(S(t), E(t), I(t), R(t))$ of system (2) satisfies

$$
\liminf _{t \rightarrow+\infty} I(t) \geq \min _{0 \leq \eta \leq \omega} m_{0} v_{22}(\eta) \exp \left(p_{2} \eta\right) .
$$

Proof. We first prove the following claim:

$$
\limsup _{t \rightarrow+\infty} I(t) \geq m_{1}
$$

where $m_{1}>0$ is sufficiently small. Suppose that (26) is not true; then there exists a $t_{1}>0$ such that $I(t)<m_{1}$ for all $t>t_{1}$. Then according to the first equation of system (2), we get

$$
\begin{array}{r}
\dot{S}(t) \geq \lambda(t)-m_{1} \beta(t) S(t)-\mu(t) S(t), \\
t \neq k T, k \in \mathbb{N}, \\
S\left(t^{+}\right)=\left(1-\theta_{k}\right) S(t), \quad t=k T, k \in \mathbb{N} .
\end{array}
$$

Consider an auxiliary system

$$
\begin{array}{r}
\dot{x}(t)=\lambda(t)-m_{1} \beta(t) x(t)-\mu(t) x(t), \\
t \neq k T, k \in \mathbb{N}, \\
x\left(t^{+}\right)=\left(1-\theta_{k}\right) x(t), \quad t=k T, k \in \mathbb{N} .
\end{array}
$$

By the comparison theorem, it following that $S(t) \geq x(t)$ holds. From Lemma 1, we see that system (28) admits a positive periodic solution $X^{*}(t)$ which is globally asymptotically stable and $\lim _{m_{1} \rightarrow 0}\left(X^{*}(t)-S^{*}(t)\right)=0$. Hence, for any $\epsilon>0$, there exits $t_{2}>t_{1}$ such that $S(t) \geq S^{*}(t)-\epsilon$ for $t>t_{2}$. Then for $t>t_{2}$, we obtain that

$$
\begin{gathered}
\dot{E}(t) \geq \frac{\beta(t)\left(S^{*}(t)-\epsilon\right) I(t)}{1+\alpha(t)\left(S^{*}(t)-\epsilon\right)}-(\mu(t)+\varepsilon(t)) E(t), \\
\dot{I}(t)=\varepsilon(t) E(t)-(\mu(t)+\gamma(t)) I(t) .
\end{gathered}
$$

If $r\left(\Phi_{F-V}(\omega)\right)>1$, choosing $\epsilon>0$, such that $r\left(\Phi_{F_{2}}(\omega)\right)>1$. By the comparison principle, there exists a positive constant $\Lambda$ such that $J(t) \geq \Lambda v_{2}(t) \exp \left(p_{2} t\right)$ where $J(t)=(E(t), I(t))^{T}$ and $p_{2}=(1 / \omega) \ln r\left(\Phi_{F_{2}}(\omega)\right)>0$, which implies that $\lim _{t \rightarrow+\infty} I(t)=+\infty$ which is a contradiction. Thus (26) holds.

In view of (26), we know that there are two possible cases.

Case $I$. $I(t) \geq m_{1}$ for all large $t$. The conclusion is evident in this case.

Case II. I $(t)$ oscillates about $m_{1}$ for all large $t$.

Set $\underline{t}\left(>t_{2}\right)$, and $\rho>0$ satisfy

$$
\begin{gathered}
I(\underline{t})=I(\underline{t}+\rho)=m_{1}, \\
I(\underline{t})<m_{1},
\end{gathered}
$$

$S(t) \geq S^{*}(t)-\epsilon$ for $\underline{t}<t<\underline{t}+\rho$.

It follows from system (2) that

$$
\begin{gathered}
\dot{E}(t) \geq \frac{\beta(t)\left(S^{*}(t)-\epsilon\right) I(t)}{1+\alpha(t)\left(S^{*}(t)-\epsilon\right)}-(\mu(t)+\varepsilon(t)) E(t), \\
\dot{I}(t)=\varepsilon(t) E(t)-(\mu(t)+\gamma(t)) I(t),
\end{gathered}
$$

for $\underline{t}<t<\underline{t}+\rho$. 


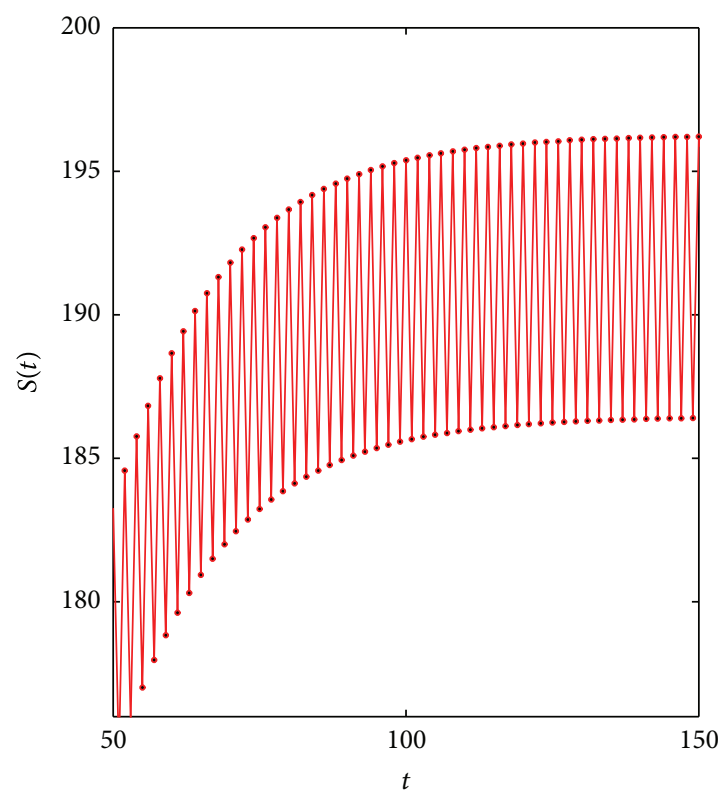

(a)

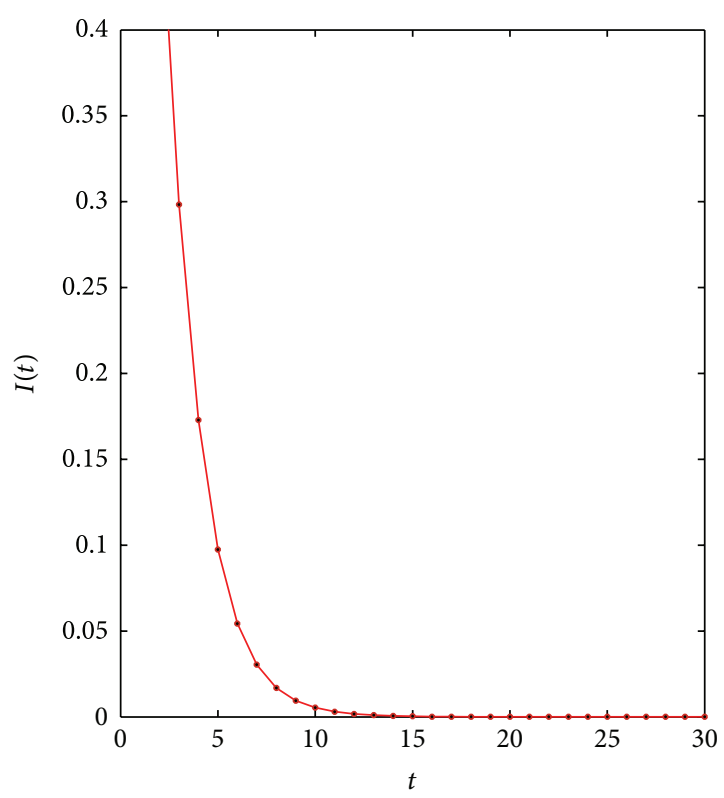

(b)

Figure 1: Dynamical behavior of system (2) with $\lambda(t)=10, \alpha(t)=0, \beta(t)=\mu(t)=0.001, \varepsilon(t)=\gamma(t)=0.8, T=\omega=1, \theta_{1}=0.1$, and $r\left(\Phi_{F-V}(\omega)\right) \simeq 0.8026$. (a) Time series of $S(t)$. (b) Time series of $I(t)$.

Denote $n_{0}=[\underline{t} / \omega]$. Since $\underline{t}$ is sufficiently large, by Lemma 5 and $I(\underline{t})=m_{1}$, we have

$$
E(\underline{t}) \geq \min \left\{\frac{m_{1}\left(\mu^{M}+\gamma^{M}\right)}{\varepsilon^{L}}, \frac{m_{1} \beta^{M} S^{M}}{\left(\mu^{L}+\varepsilon^{L}\right)\left(1+\alpha^{L} S^{M}\right)}\right\} .
$$

Then, by the comparison principle, there exists a positive $m_{0}$ such that

$$
\begin{array}{r}
J(t) \geq m_{0} v_{2}\left(t-n_{0} \omega\right) \exp \left(p_{2}\left(t-n_{0} \omega\right)\right), \\
\underline{t}<t<\underline{t}+\rho,
\end{array}
$$

where $m_{0}$ is independent of the choice of the interval $[\underline{t}, \underline{t}+\rho]$. Thus we have that

$$
\begin{aligned}
I(t) & \geq m_{0} v_{22}\left(t-n_{0} \omega\right) \exp \left(p_{2}\left(t-n_{0} \omega\right)\right) \\
& \geq \min _{0 \leq \eta \leq \omega} m_{0} v_{22}(\eta) \exp \left(p_{2} \eta\right), \quad \underline{t}<t<\underline{t}+\rho .
\end{aligned}
$$

Since the kind of interval $[\underline{t}, \underline{t}+\rho]$ is chosen in an arbitrarily way (we only need $t$ to be sufficiently large) and $\min _{0 \leq \eta \leq \omega} m_{0} v_{22}(\eta) \exp \left(p_{2} \eta\right)$ is independent of the positive solution of system (2), we have proved that any solution of system of (2) satisfies $I(t) \geq \min _{0 \leq \eta \leq \omega} m_{0} v_{22}(\eta) \exp \left(p_{2} \eta\right)$, for sufficiently large $t$. The proof is completed.

The following are two immediate corollaries of Theorem 6.

Corollary 7. The diseases-free periodic solution of (2) is unstable when $r\left(\Phi_{F-V}(\omega)\right)>1$.
It follows from Lemma 5 and Theorem 6 that the following result holds.

Theorem 8. If $r\left(\Phi_{F-V}(\omega)\right)>1$, then the system (2) is permanent.

\section{Discussion and Numerical Results}

In this paper, we have established and studied a nonautonomous SEIR endemic model with saturation incidence and pulse vaccination. From the theoretically analysis, we obtain a threshold $r\left(\Phi_{F-V}(\omega)\right)$, and there exists a globally asymptotically stable disease-free periodic solution if $r\left(\Phi_{F-V}(\omega)\right)<1$ (see Theorem 4). However, when $r\left(\Phi_{F-V}(\omega)\right)>1$, the stability of disease-free periodic solution is lost, and system (2) is permanent (see Theorem 6). According to [16] we can define the spectral radius of the $\Phi_{F-V}(\omega)$ as the basic reproduction ratio

$$
R_{0}:=r\left(\Phi_{F-V}(\omega)\right)
$$

for system (2). It is worthwhile for us to study how to define the basic reproduction ratio for a general system with pulse interval interruption.

To illustrate the mathematical results, we investigate future by using numerical simulations. Now consider the following two examples which Theorems 4 and 6 are satisfied.

Consider the following set of system $(2): \lambda(t)=10, \alpha(t)=$ $0, \beta(t)=\mu(t)=0.001, \varepsilon(t)=\gamma(t)=0.8, T=\omega=1$, and $\theta_{1}=0.1$. 


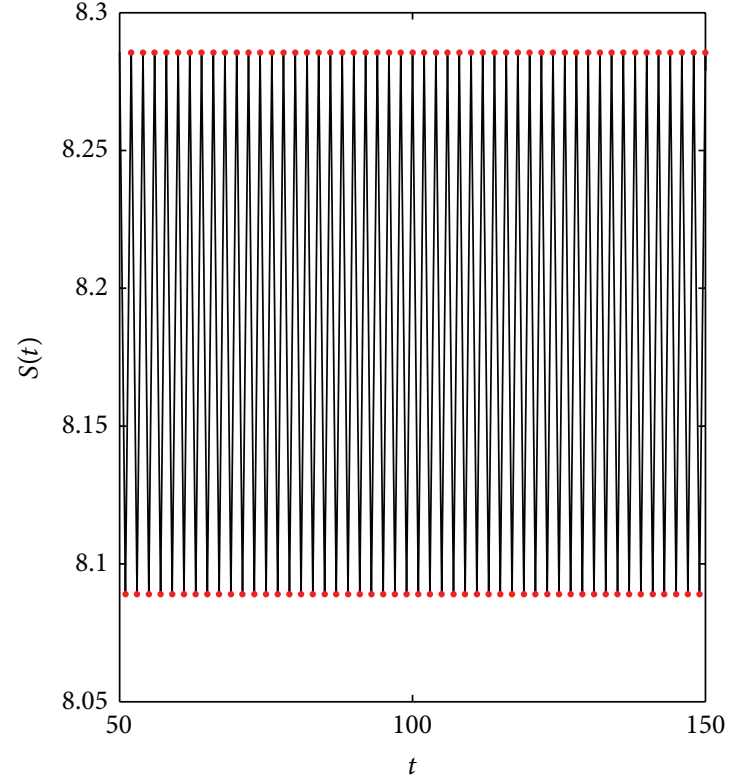

(a)

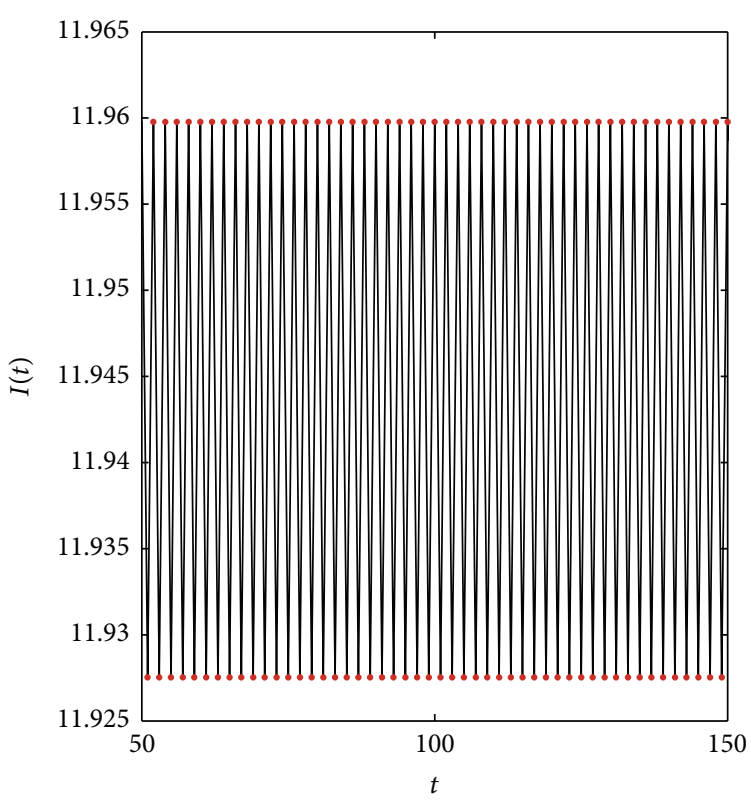

(b)

Figure 2: Dynamical behavior of system (2) with $\lambda(t)=10, \alpha(t)=0, \beta(t)=0.1, \mu(t)=0.0001, \varepsilon(t)=\gamma(t)=0.8, T=\omega=1, \theta_{1}=0.1$, and $r\left(\Phi_{F-V}(\omega)\right) \simeq 1.1527$. (a) Time series of $S(t)$. (b) Time series of $I(t)$.

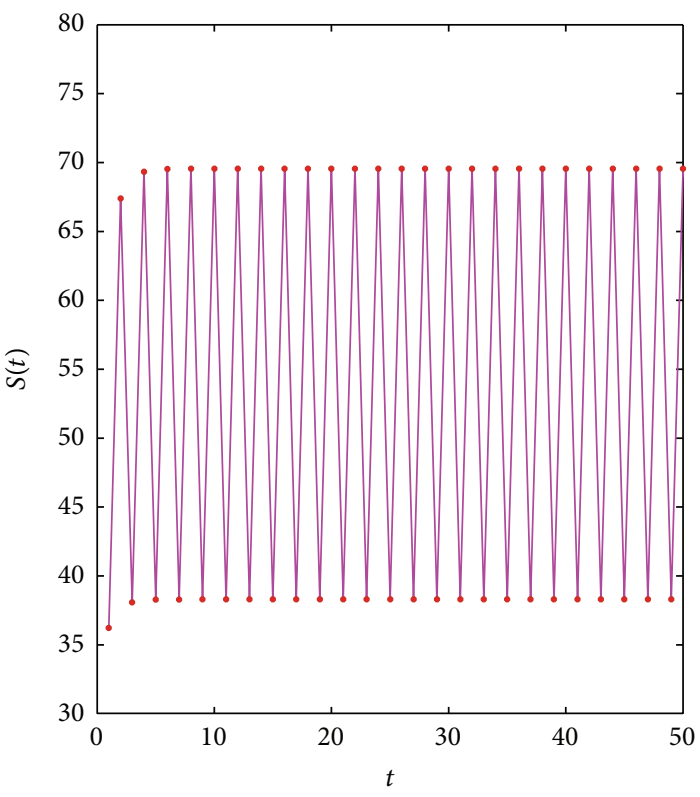

(a)

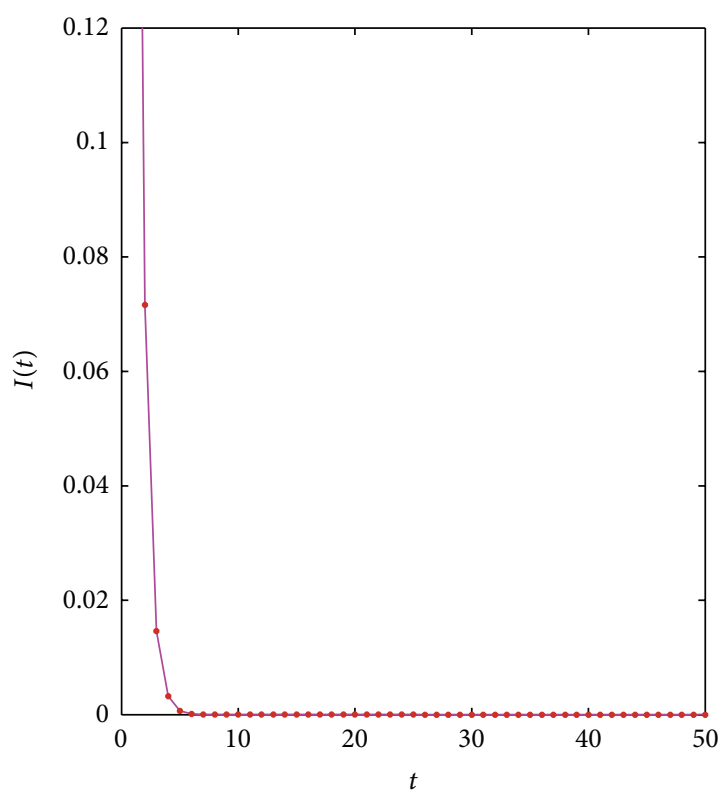

(b)

Figure 3: Dynamical behavior of system (2) with $\beta(t)=0.005(\sin t+2), \lambda(t)=10, \alpha(t)=0.01, \mu(t)=0.1, \gamma(t)=0.1, \varepsilon(t)=0.8, \omega=2 T=2 \pi$, and $\theta_{1}=\theta_{2}=0.9$. (a) Time series of $S(t)$. (b) Time series of $I(t)$.

We have

$$
\begin{aligned}
S^{*}(t)= & e^{-0.001 t} \frac{1000-1000 e^{-0.001 t}}{1-e^{-0.001 t}} \\
& +10000-10000 e^{-0.001 t}, \\
n \omega< & t \leq(n+1) \omega, \quad n \in \mathbb{N} .
\end{aligned}
$$

By a approximation method, we obtain $r\left(\Phi_{F-V}(\omega)\right) \simeq 0.8026$. Hence, by Theorem 4 , the disease-free periodic solution $\left(S^{*}(t), 0,0, R^{*}(t)\right)$ is globally asymptotically stable. The disease will die out. Numerical simulation illustrates this fact (see Figure 1).

The following choice of parametric values: $\lambda(t)=10$, $\alpha(t)=0, \beta(t)=0.1, \mu(t)=0.0001, \varepsilon(t)=\gamma(t)=0.8$, $T=\omega=1$, and $\theta_{1}=0.1$. 


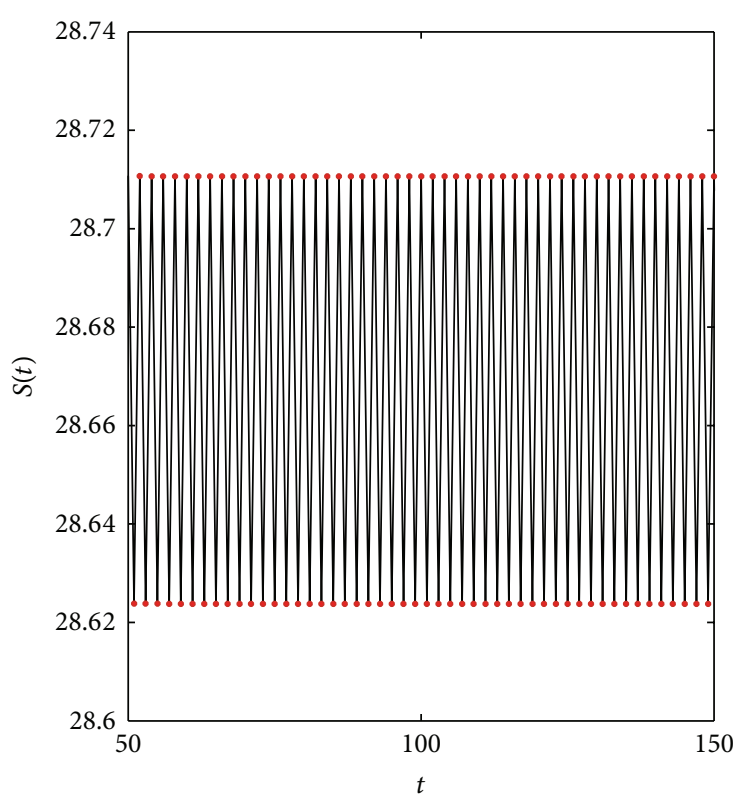

(a)

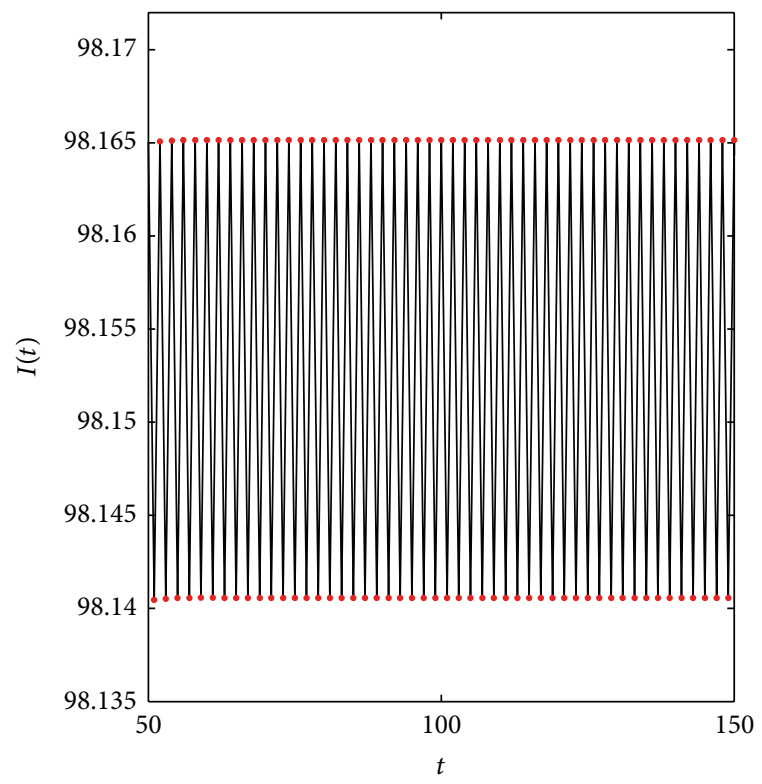

(b)

Figure 4: Dynamical behavior of system (2) with $\beta(t)=0.005(\sin t+2), \lambda(t)=10, \alpha(t)=0.01, \mu(t)=0.1, \gamma(t)=0.1, \varepsilon(t)=0.8, \omega=2 T=2 \pi$, and $\theta_{1}=\theta_{2}=0.01$. (a) Time series of $S(t)$. (b) Time series of $I(t)$.

We have

$$
\begin{aligned}
S^{*}(t)= & e^{-0.0001 t} \frac{10000-10000 e^{-0.0001 t}}{1-e^{-0.0001 t}} \\
& +100000-100000 e^{-0.0001 t}, \\
& n \omega<t \leq(n+1) \omega, \quad n \in \mathbb{N} .
\end{aligned}
$$

By a approximation method, we obtain $r\left(\Phi_{F-V}(\omega)\right) \simeq 1.1527$. Clearly, in view of Theorem 6, the disease will become endemic (see Figure 2).

It is interesting to examine how the pulse vaccination affects the transmission of the disease. Fix the parameters of system (2) as follows: $\beta(t)=0.005(\sin t+2), \lambda(t)=10, \alpha(t)=$ $0.01, \mu(t)=0.1, \gamma(t)=0.1, \varepsilon(t)=0.8$, and $\omega=2 T=2 \pi$.

Numerical results shown in Figure 3 imply that the disease will be eradicated if we set $\theta_{1}=\theta_{2}=0.9$. In contrast, the disease will become endemic shown in Figure 4 if set $\theta_{1}=\theta_{2}=0.01$. Obviously, the pulse vaccination plays an important role in the system.

\section{Conflict of Interests}

The authors declare that there is no conflict of interests regarding the publication of this paper.

\section{Acknowledgments}

This work is supported by Key Laboratory of Biologic Resources Protection and Utilization of Hubei Province (PKLHB1302), the Soft Science Research Project of Hubei Province (2012GDA01309), Natural Science Foundation of
Education Committee of Hubei Province (B2013073), and Key Discipline of Hubei Province-Forestry.

\section{References}

[1] G. Jiang and Q. Yang, "Periodic solutions and bifurcation in an SIS epidemic model with birth pulses," Mathematical and Computer Modelling, vol. 50, no. 3-4, pp. 498-508, 2009.

[2] T. Zhang and Z. Teng, "Pulse vaccination delayed SEIRS epidemic model with saturation incidence," Applied Mathematical Modelling, vol. 32, no. 7, pp. 1403-1416, 2008.

[3] C. Zhang, Y. Zhao, and Y. Wu, "An impulse model for computer viruses," Discrete Dynamics in Nature and Society, vol. 2012, Article ID 260962, 13 pages, 2012.

[4] T. Zhang and Z. Teng, "Extinction and permanence for a pulse vaccination delayed SEIRS epidemic model," Chaos, Solitons \& Fractals, vol. 39, no. 5, pp. 2411-2425, 2009.

[5] C. Zhang, Y. Zhao, and Y. Wu, "An impulse dynamic model for computer worms," Abstract and Applied Analysis, vol. 2013, Article ID 286209, 8 pages, 2013.

[6] X. Meng, J. Jiao, and L. Chen, "Two profitless delays for an SEIRS epidemic disease model with vertical transmission and pulse vaccination," Chaos, Solitons \& Fractals, vol. 40, no. 5, pp. 21142125, 2009.

[7] T. Zhang, J. Liu, and Z. Teng, "Existence of positive periodic solutions of an SEIR model with periodic coefficients," Applications of Mathematics, vol. 57, no. 6, pp. 601-616, 2012.

[8] X. Ji, Y. Pei, and C. Li, "Two patterns of recruitment in an epidemic model with difference in immunity of individuals," Nonlinear Analysis: Real World Applications, vol. 11, no. 3, pp. 2078-2090, 2010.

[9] H.-F. Huo and L.-X. Feng, "Global stability of an epidemic model with incomplete treatment and vaccination," Discrete 
Dynamics in Nature and Society, vol. 2012, Article ID 530267, 14 pages, 2012.

[10] X. Liu and P. Stechlinski, "Pulse and constant control schemes for epidemic models with seasonality," Nonlinear Analysis: Real World Applications, vol. 12, no. 2, pp. 931-946, 2011.

[11] C. Sun and Y.-H. Hsieh, "Global analysis of an SEIR model with varying population size and vaccination," Applied Mathematical Modelling, vol. 34, no. 10, pp. 2685-2697, 2010.

[12] M. de la Sen, A. Ibeas, and S. Alonso-Quesada, "Observerbased vaccination strategy for a true mass action SEIR epidemic model with potential estimation of all the populations," Discrete Dynamics in Nature and Society, vol. 2011, Article ID 743067, 19 pages, 2011.

[13] W. Wang, J. Xin, and F. Zhang, "Persistence of an SEIR model with immigration dependent on the prevalence of infection," Discrete Dynamics in Nature and Society, vol. 2010, Article ID 727168, 7 pages, 2010.

[14] Z. Bai and Y. Zhou, "Existence of two periodic solutions for a non-autonomous SIR epidemic model," Applied Mathematical Modelling, vol. 35, no. 1, pp. 382-391, 2011.

[15] N. Bacaër and S. Guernaoui, "The epidemic threshold of vector-borne diseases with seasonality. The case of cutaneous leishmaniasis in Chichaoua, Morocco," Journal of Mathematical Biology, vol. 53, no. 3, pp. 421-436, 2006.

[16] W. Wang and X.-Q. Zhao, "Threshold dynamics for compartmental epidemic models in periodic environments," Journal of Dynamics and Differential Equations, vol. 20, no. 3, pp. 699-717, 2008.

[17] Y. Nakata and T. Kuniya, "Global dynamics of a class of SEIRS epidemic models in a periodic environment," Journal of Mathematical Analysis and Applications, vol. 363, no. 1, pp. 230237, 2010.

[18] T. Zhang and Z. Teng, "On a nonautonomous SEIRS model in epidemiology," Bulletin of Mathematical Biology, vol. 69, no. 8, pp. 2537-2559, 2007.

[19] V. Lakshmikantham, D. D. Baĭnov, and P. S. Simeonov, Theory of Impulsive Differential Equations, vol. 6, World Scientific Publishing, Singapore, 1989.

[20] J. J. Kim, M. Brisson, W. J. Edmunds, and S. J. Goldie, "Modeling cervical cancer prevention in developed countries," Vaccine, vol. 26, no. 10, pp. K76-K86, 2008. 


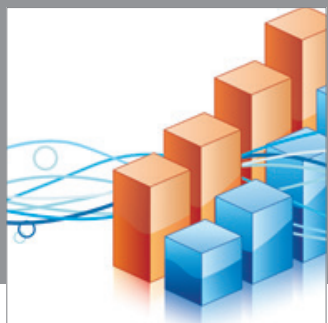

Advances in

Operations Research

mansans

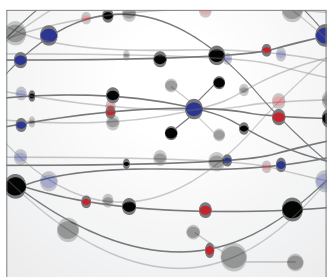

The Scientific World Journal
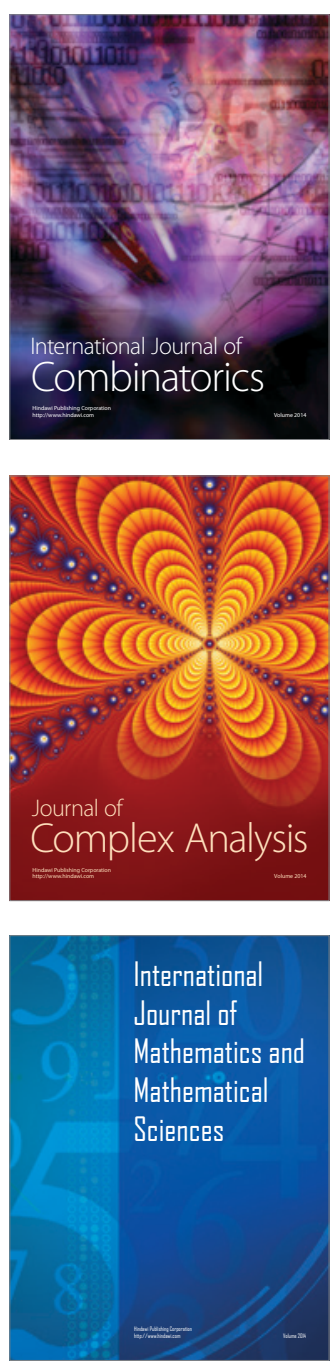
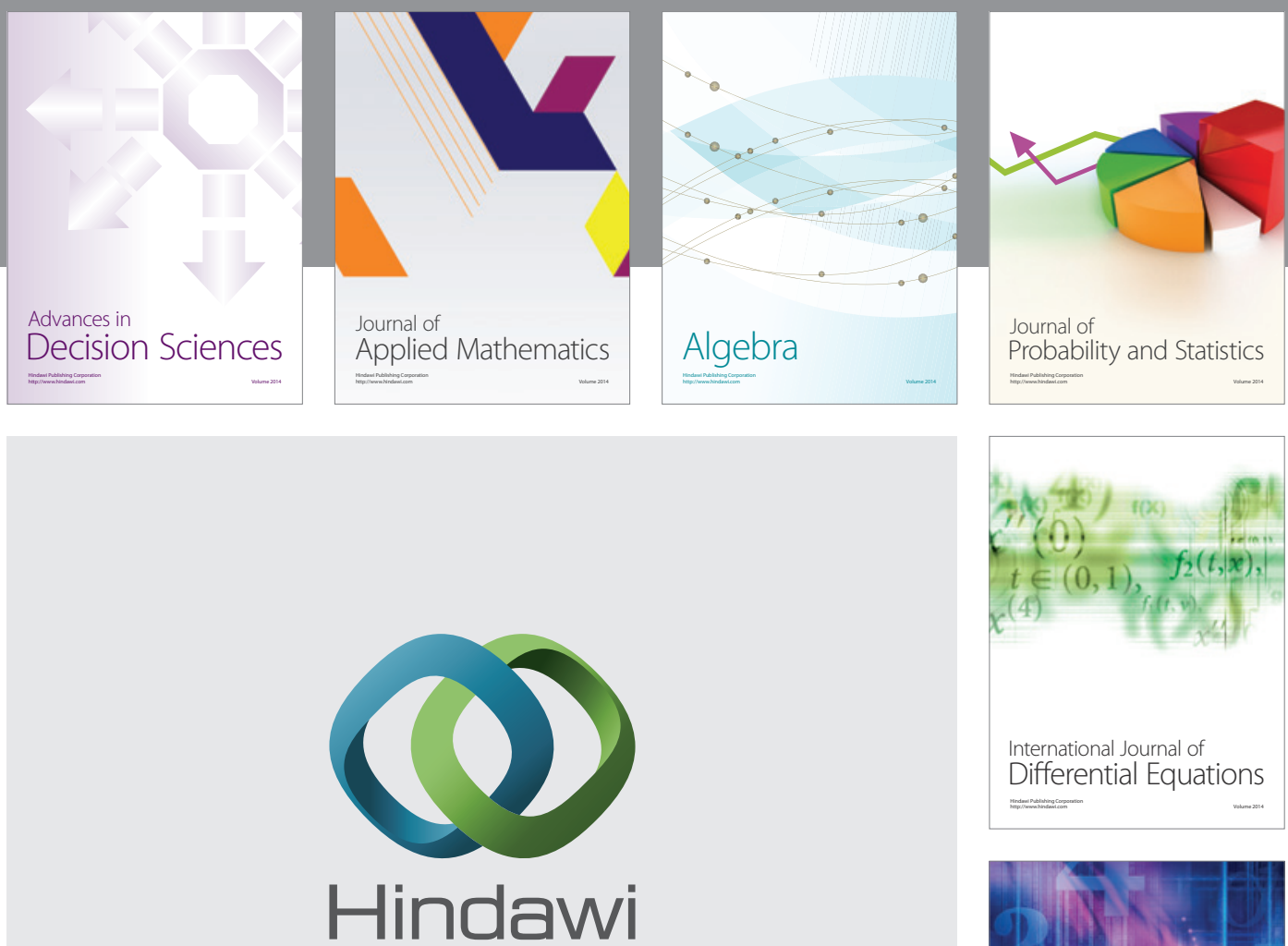

Submit your manuscripts at http://www.hindawi.com
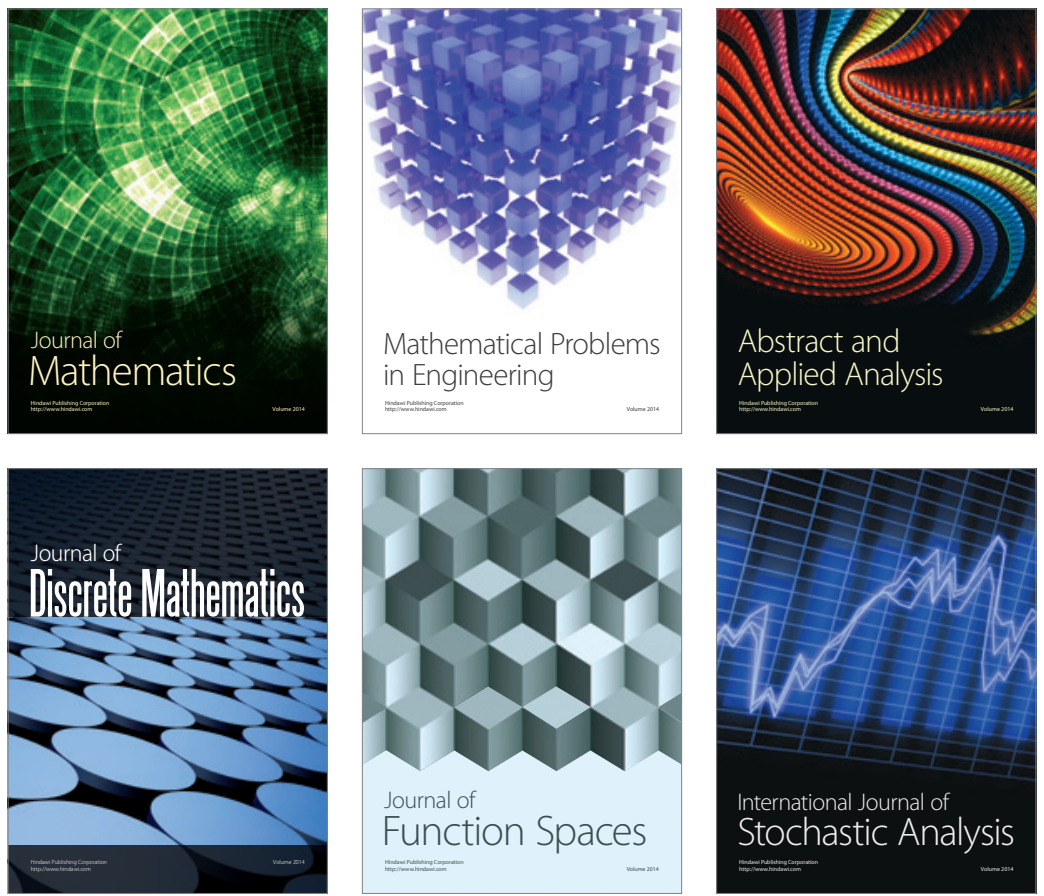

Journal of

Function Spaces

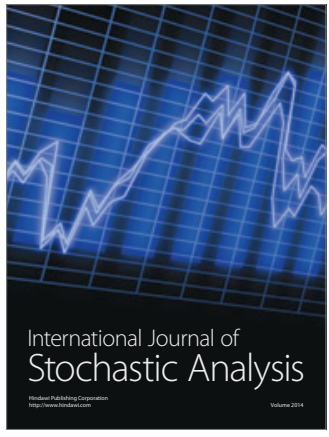

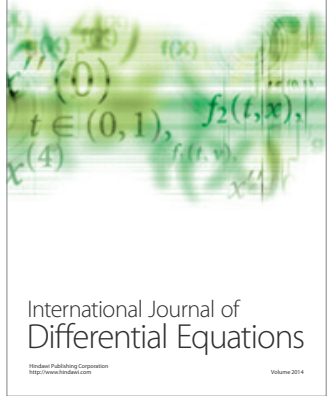
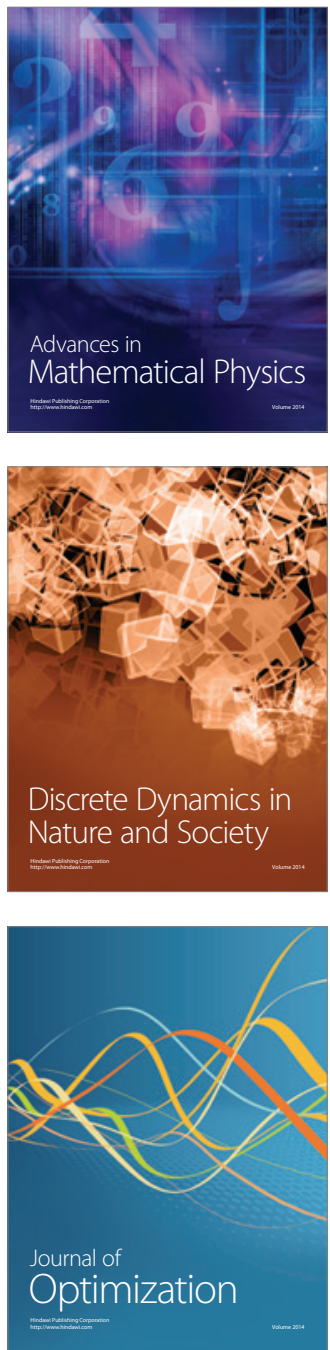